\title{
FACTITIOUS DISORDER PRESENTING WITH APPARENT PSYCHOTIC SYMPTOMS: CHALLENGES FOR CLINICAL ASSESSMENT
}

R. Fernandes, S. Vilas Boas Garcia, T. Teodoro, M. Martins

Centro Hospitalar Psiquiátrico de Lisboa, Lisboa, Portugal

\section{INTRODUCTION}

Factitious disorder (FD), firstly described as Munchausen syndrome by Asher is characterized by falsification of physical or psychological signs or symptoms that are intentionally produced or feigned in the absence of apparent external incentives and associated with identified deception. FD is fairly uncommon, but likely to be underdiagnose and generate high costs in health care facilities. We present a case of factitious disorder presenting with apparent psychotic symptoms.

\section{CASE REPORT}

27 year-old woman, single. No previous history of mental illness.

Admitted in the psychiatric emergence department reporting florid psychotic symptoms lasting for several months. She reported hearing several different voices, described vaguely as being inside her head, sometimes giving her commands, poorly characterized. Additionally she reported to start having visions, namely to see blood dripping from water taps, and smelling strange smells in food, providing few details. She also described the presence of more intense "crisis", witnessed by other people, where she would become agitated with incoherent speech, hearing voices, having visions and with a vague persecutory feeling, although poorly recorded. She reported that these phenomena made her feel very anxious. She had consulted a psychiatrist who prescribed her injectable paliperidone $50 \mathrm{mg}$, which she took only once, with no symptomatic improvement.

She was admitted for inpatient management. Laboratory examinations and cranial CT were normal.

At mental examination she showed an indifferent attitude, laconic and evasive speech, inconsistent affect, describing feelings of anxiety and anguish due to hallucinatory activity with an apparent vague expression and poor affective resonance. No apparent hallucinatory activity during the interview. Form and content of thought with no alterations. No memory or orientation defect was detected. Insight was preserved.

After family interview and investigation of clinical records from other hospitals, it was found that she had a long history of soughting treatment at various hospitals presenting with variable medical symptoms, a remarkable number of consultations and several hospitalizations; it was discovered falsification of clinical data and selfinduced findings and a diagnosis of factitious disorder had already been considered.

\section{DISCUSSION}

Although FD has been officially recognized in the DSM for more than 30 years, it's diagnostic validity and classification is still controversial. History of multiple hospitalizations, symptoms with unknown etiology, exaggerated or fake histories, inconsistency between clinical picture and medical findings are some common characteristics observed in the patients with FD. The differential diagnosis includes a variety of medical and psychiatric conditions. Specifically, differentiating true psychosis from FD presenting with apparent psychotic symptoms can be quite challenging. The decision is made by a thorough evaluation of the symptoms consistency, the person's medical history and with corroboration from other people. In this case, the florid psychotic symptoms described in an inconsistent and evasive manner, the incongruence between the described psychic state and the affective expression, allied with the discovery of misleading personal history details, the information provided by family members and her medical history with a diagnosis of factitious disorder already considered supported the diagnosis of FD with psychotic symptoms. There is limited information except for few case reports about FD with psychological symptoms in the literature. Suspicion of FD is the key point for diagnosis. A multidisciplinary approach is required in the diagnostic and treatment processes. 